\title{
Bilan scientifique du colloque FLOODRisk 2016 à Lyon, du 17 au 21 octobre 2016
}

\begin{abstract}
Michel LANG
Irstea, Unité de recherche Hydrologie-Hydraulique, centre de Lyon-Villeurbanne

RÉSUMÉ. - La conférence FLOODrisk 2016 a réuni plus de 500 participants d'une trentaine de pays autour de la gestion du risque d'inondation. Les actes du colloque avec 254 papiers scientifiques ou techniques sont librement accessibles sur Internet. Le présent article dresse une rapide synthèse des principaux éléments abordés lors de la conférence autour de six grands thèmes : 1 / estimation de l'aléa ; 2 / estimation des conséquences humaines et économiques ; 3 / estimation du risque d'inondation ; 4/ actions de prévention ; 5/ gestion des crises ; 6/ outils de gouvernance. Une sélection de 7 articles en anglais a été retenue pour publication dans la revue La Houille Blanche sur les retours d'expérience qui ont pu être produits en Europe sur la mise en œuvre de la Directive Inondation. Nous en résumons ici les éléments marquants.
\end{abstract}

Mots-clés : Directive Inondation, FloodRisk 2016, Prévention, Risque inondation

\section{Scientific conclusions of FLOODRisk 2016 conference, Lyon, 17-21 October 2016}

\begin{abstract}
The FLOODrisk 2016 conference brought together over 500 participants from around thirty countries around flood risk management. The proceedings of the colloquium with 254 scientific or technical papers are freely available on the Internet. This article provides a brief summary of the main elements discussed at the conference on six main topics: 1 / characterizing the flood hazard; 2 / characterizing the consequences; 3 / characterizing the flood risk; 4 / risk reduction and management; 5 / flood event management; 6 / decision making, policy and governance. A selection of 7 articles in English was selected for publication in the journal La Houille Blanche on the feedback that could be produced in Europe on the implementation of the Flood Directive. Here we summarize the salient features.
\end{abstract}

Key-words: Flood Directive, FloodRisk 2016, Flood risk prevention

\section{ORGANISATION DU COLLOQUE}

La conférence quadriennale FLOODrisk 2016 à Lyon a été la troisième d'une série consacrée à la gestion du risque d'inondation, après celles d'Oxford en 2008 et de Rotterdam en 2012. FLOODrisk 2008 a été une initiative du projet FLOODsite (2005-2008) coordonné par HR Wallingford et financé dans le cadre du sixième Programme Cadre de Recherche et Développement de 1'Union Européenne, avec 37 partenaires scientifiques et un budget de 14 millions d'euros. L'idée initiale de cette conférence était d'organiser un espace d'échange entre chercheurs et acteurs de la gestion du risque d'inondation : politiques et élus, gestionnaires opérationnels, bureaux d'étude, industriels, associations... FLOODrisk 2008 a permis de faire connaître les récents développements et innovations autour de la gestion du risque d'inondation. FLOODrisk 2012 et FLOODrisk 2016 ont poursuivi dans la même direction et ont permis d'avoir tous les quatre ans un nouveau point sur l'évolution des techniques et des pratiques sur le risque d'inondation.

Cette troisième conférence FLOODrisk 2016 a réuni plus de 500 participants d'une trentaine de pays, principalement d'Europe mais aussi des Etats-Unis (5\%), Brésil (2\%) et Japon (2\%). Les quatre pays les plus représentés étaient la France (33\%), le Royaume-Uni (18\%), les Pays-Bas (10\%) et l'Allemagne $(10 \%)$. On note une bonne diversité sur l'origine professionnelle des participants : université (31\%), structures privées $(19 \%)$, autorités publiques $(18 \%)$, centres de recherche $(16 \%)$, bureaux d'étude $(10 \%)$, assureurs $(4 \%)$, organisations non gouvernementales $(2 \%)$.
L'organisation du colloque a bénéficié du soutien financier du Ministère de l'Environnement, de l'Energie et de la Mer, et a été coordonnée par un consortium autour de 4 organismes (Irstea, Deltares, Wallingford, Samui). Elle s'est appuyée sur un comité scientifique constitué d'une soixantaine de référents (16 pays) et sur un comité consultatif international ( 9 pays) chargé de faire le lien entre chercheurs et opérationnels. A noter l'aide financière de 6 sponsors et la présence pendant le colloque d'une quarantaine de stands d'exposition (organismes scientifiques ou techniques, bureaux d'étude, distributeurs de matériel technique).

\section{SESSIONS PARALLÈLES}

Quarante sessions parallèles ont été organisées autour de six grands thèmes liés au risque d'inondation : 1/ estimation de l'aléa ; 2/ estimation des conséquences humaines et économiques ; 3/ estimation du risque d'inondation ; 4/ actions de prévention ; 5/ gestion des crises ; 6/ outils de gouvernance. Au total, près de 400 résumés ont été examinés par le comité scientifique puis répartis en 200 communications orales et 100 posters. Ils ont fait l'objet de la publication d'actes distribués pendant la conférence sous forme numérique, avec 254 papiers scientifiques ou techniques révisés par le comité scientifique et référencés avec un identifiant numérique doi. L'ensemble des articles peut librement être consulté et téléchargé :

http://www.e3s-conferences.org/articles/e3sconf/abs/2016/02/ contents/contents.html 


\section{II.1. Estimation de l'aléa}

Si l'hydrologie et l'hydraulique sont souvent considérées aujourd'hui comme des sciences « matures », elles font toujours l'objet d'importants développements qui s'appuient sur l'augmentation de la puissance informatique et l'amélioration des techniques de calcul numérique. Ces progrès permettent d'avoir une meilleure résolution spatiale, d'inclure dans la modélisation une multiplicité de processus élémentaires et de développer des approches probabilistes adaptées à la représentation des incertitudes. On notera la complémentarité des approches reposant sur les mesures expérimentales en rivière ou bassin versant, l'utilisation de laboratoires expérimentaux et la modélisation numérique pour in fine mieux représenter et cartographier les zones inondées. Le fait de pouvoir produire des animations plus réalistes sur l'inondation de zones sensibles contribue à améliorer la communication de résultats techniques auprès du public. Une excellente communication «Applying emulators for improved flood risk analysis » de Sajni Malde (HR Wallingford) et al. a obtenu le prix de la meilleure contribution d'un jeune auteur. Elle porte sur l'utilisation d'un émulateur Gaussien permettant de réduire significativement le nombre d'itérations dans des simulations Monte-Carlo, qui sont aujourd'hui à la base de nombreux calculs sur l'aléa.

D'importants progrès ont été faits via l'analyse probabiliste multivariée qui permet de combiner plusieurs types d'aléa pour le risque de submersion marine (surcote de pleine mer, marée, propagation sur la côte) ou via les analyses régionales, qui combinent l'information issue d'une série de sites de mesure et la transfèrent en un point quelconque du territoire. On notera une bonne expérience du Royaume-Uni dans ce type d'approches. Des analyses prospectives ont porté sur l'impact du changement climatique sur l'aléa inondation, en particulier pour les submersions marines sur le littoral en Europe.

La question de la stabilité des ouvrages de défense hydraulique a fait l'objet de nombreux échanges sur la modélisation des dégradations et des ruptures d'une digue, les retours d'expérience issus d'expérimentations de terrain pour tester différentes structures et mieux comprendre les processus en jeu, les études de danger pour vérifier la sécurité des structures existantes, le développement de systèmes d'information pour suivre l'état des digues.

\section{II.2. Estimation des conséquences humaines et économiques}

Une bonne connaissance du risque de mortalité passe par une collecte systématique d'informations sur les inondations passées pour mieux comprendre les circonstances de décès et mieux modéliser le type de comportement des différents acteurs. Ces éléments peuvent être exploités pour arbitrer entre l'évacuation de personnes en danger et leur mise en sécurité in situ (cf. risques liés au déplacement). La prise de décision doit pouvoir s'appuyer sur des approches simplifiées permettant de repérer rapidement les zones de fort danger. Un des enjeux est de progresser dans la façon de prendre en compte les incertitudes.

L'estimation des dommages induits par une inondation passée et la modélisation des conséquences d'une série d'inondations de référence à venir passent par la collecte de données post-catastrophe pour mieux valider les fonctions d'endommagement. Des efforts sont encore nécessaires pour harmoniser la collecte de données et mieux partager ces informations. Les travaux en cours ont porté sur l'amélioration de la cartographie des enjeux exposés et la mise au point de fonctions d'endommagement par typologie d'enjeux. Des illustrations de ces approches ont été présentées dans différents pays (Italie, USA, France, RoyaumeUni, Allemagne, Turquie).

Un point délicat concerne l'évaluation des effets dominos liés à la propagation amont-aval d'une onde de crue et à la vulnérabilité de systèmes complexes constitués d'une multitude de réseaux élémentaires dépendants (énergie, eau, transport, télécommunication). Une bonne appréciation de ce type de conséquences passe par un recensement des infrastructures critiques, la connaissance de leur vulnérabilité et interdépendances, et une planification de mesures appropriées pour améliorer la résilience des systèmes. De nombreux cas d'étude ont été présentés (France, Pays-Bas, Allemagne, Royaume-Uni, Italie, Finlande, Etats-Unis, Tchéquie, Irlande, Pologne).

\section{II.3. Estimation du risque d'inondation}

De nombreuses analyses de risques ont été présentées, à l'échelle nationale ou de grands bassins versants, et pour différents enjeux : réseau routier stratégique, réseau ferroviaire, littoral... Plusieurs communications ont porté sur la façon de représenter les vulnérabilités physiques et sociales et sur la signification du terme de résilience. Des cas d'application ont illustré ces différentes notions, avec des outils de modélisation plus ou moins détaillés et des approches participatives auprès des acteurs concernés. Deux exemples d'enquête de terrain (France, Royaume-Uni) ont montré la difficulté à développer une réelle culture du risque, avec souvent l'absence de dispositif pérenne approprié et la volonté d'oublier les traumatismes d'événements récents d'inondation.

\section{II.4. Actions de prévention}

La principale mesure de prévention du risque d'inondation utilisée dans le passé consistait à mettre en place des ouvrages de protection (digues et barrages). Des bilans ont été présentés sur cette politique et sur les actions à mener pour garantir la sécurité de ces ouvrages. On peut noter de nouvelles techniques de surveillance (Lidar, drones) qui permettent un meilleur suivi des ouvrages, mais qui nécessitent le traitement d'une grande quantité d'information.

Une évolution majeure réside aujourd'hui dans la prise de conscience d'intégrer dans le dispositif de prévention des mesures non directement liées à la réduction de l'aléa : maintien d'un espace de divagation des cours d'eau dans le lit majeur et de zones d'expansion, intérêt de conserver des zones végétalisées en bordure des cours d'eau, réduction de la vulnérabilité et amélioration de la résilience, développement de la culture et de la mémoire sur le risque. De nouveaux standards ont été retenus pour la définition de bâtiments résistants-résilients aux inondations, et des propositions ont été faites pour réduire la vulnérabilité des zones urbaines. Le bilan sur la réelle implication-compréhension de la population vis-à-vis du risque d'inondation reste très mitigé (cf. enquête en France à Orléans), même si des initiatives intéressantes ont été présentées au RoyaumeUni, avec la mise en place de groupes associatifs impliqués dans la gestion du risque d'inondation, la mise en ligne de témoignages numériques (audio, vidéo) post-catastrophes et un travail de recherche pour améliorer la participation du public. Le développement de la mémoire du risque passe par des actions de mise à disposition d'informations sur les inondations passées et une meilleure appropriation de ces éléments par la société. 


\section{II.5. Gestion des crises}

De nombreux développements techniques sont réalisés pour améliorer la prévision des inondations et aider à une meilleure gestion des crises. Le cas des bassins à crue rapide reste difficile avec un très faible délai d'anticipation vu les temps de réaction et l'importance de bien prévoir de façon quantitative et spatialement distribuée les pluies à venir. Les développements actuels visent à modéliser les différentes sources d'incertitude et à produire une prévision probabiliste qui permet d'avoir une information sur la fiabilité des prévisions. On notera une évolution sur l'objet de la prévision, pour passer de la prévision de cotes ou débits au droit de stations hydrométriques à une prévision des zones inondées, à l'échelle d'abord de tronçons du réseau hydrométrique puis de façon distribuée sur l'ensemble du territoire. Plusieurs applications ont porté sur le couplage de modèles de prévision hydrologique avec des modèles d'impact, pour prédire les conséquences des inondations et aider les services opérationnels à orienter prioritairement leurs interventions en période de crise. La mise en place de zones de refuge à l'intérieur de bâtiments exposés ou à proximité immédiate peut être une alternative intéressante à l'évacuation de la population. Des dispositifs amovibles (sacs de sable, structures gonflables) sont proposés pour renforcer temporairement des digues dont la stabilité apparaît comme menacée.

Le processus de reconstruction après une catastrophe constitue une opportunité pour revoir les schémas de développement du territoire, avec une urbanisation prenant davantage en compte le risque inondation. Des éléments de diagnostic ont été apportés sur les mécanismes financiers de reconstruction dans différents pays, sur la façon dont les territoires ont été reconstruits après des inondations catastrophiques, et sur l'implication des citoyens dans ce processus.

\section{II.6. Outils de gouvernance}

Plusieurs présentations ont porté sur la mise en application de la Directive Inondation dans différents pays européens, et plus généralement sur les outils de gouvernance du risque d'inondation utilisés en Europe et aux Etats-Unis. Des programmes nationaux d'ampleur ont été mis en place aux Pays-Bas et au Royaume-Uni, avec de forts enjeux économiques et une hiérarchisation des actions en fonction des dommages potentiels. Les approches coût-bénéfice apportent des éléments utiles à la prise de décision. L'implication du public et des acteurs locaux reste insuffisante, du fait d'une mauvaise connaissance du risque d'inondation et par voie de conséquence un faible intérêt à se mobiliser, au-delà de la phase assez courte qui succède à un événement catastrophique. La question de l'équité des mesures de gestion du risque d'inondation a été abordée par plusieurs auteurs et ne doit pas être négligée dans les approches techniques et économiques. Le rôle important des assurances a été souligné, avec des interrogations sur la façon de responsabiliser les sinistrés, des enquêtes pour mieux comprendre les facteurs favorisant la prise de contrat d'assurance et le besoin pour les assureurs de disposer d'informations sur la résilience des bâtiments.

Les analyses prospectives montrent une forte augmentation du risque d'inondation à venir au cours du XXIe siècle, en fonction de scénarios sur le réchauffement du climat et sur l'évolution démographique et des enjeux exposés en zone inondable. La recherche de scénarios adaptatifs de gestion du risque d'inondation, à réactualiser régulièrement apparaît comme la piste à suivre.

\section{PUBLICATIONS D'ARTICLES RELATIFS À LA MISE EN GEUVRE DE LA DIRECTIVE EUROPÉENNE SUR LES INONDATIONS}

Le colloque FLOODrisk 2016 a fait l'objet d'un atelier spécifique consacré à la mise en œuvre de la Directive Inondation (DI) dans les différents pays européens, et de 7 communications orales sur le sujet. Comme le premier cycle de 6 ans (2010-2015) d'implémentation de la DI venait de se terminer, il nous a semblé intéressant de faire partager au sein de la revue La Houille Blanche les différents retours d'expérience qui ont pu être produits.

La DI a été mise en œuvre dans les différents pays européens, en suivant généralement une approche de croisement de l'aléa inondation avec des cartes d'enjeu et de vulnérabilité. Elle conduit à évaluer le niveau actuel du risque inondation en termes de conséquences économiques, de personnes exposées et d'impacts environnementaux. Des mesures de prévention, protection, préparation, sensibilisation et résilience sont ensuite proposées. Au niveau français, Morel et al. (2017) décrivent la procédure retenue, en cohérence avec les outils existants de gestion du risque et les différentes structures territoriales de gestion du risque. Dupray et al. (2017) présentent les principales leçons tirées sur le bassin de la Seine pour la préparation des plans de gestion, que ce soit au niveau technique (cartes d'aléa et de risque, mesures préconisées de gestion du risque), des échanges et de la concertation avec les différents acteurs concernés, et au niveau des évolutions législatives concomitantes sur les autorités de gestion territoriale.

Verbeke et Devroede (2017) expliquent la façon dont les différentes mesures de gestion du risque d'inondation ont été évaluées et priorisées dans les Flandres (Belgique). Par ailleurs, des hypothèses-scénarios sur l'impact du changement climatique et l'occupation future du territoire ont été testées. En Autriche, Neuhold (2017a) souligne l'importance d'une bonne concertation entre les différents acteurs concernés, mentionne l'intérêt de prendre en compte un risque résiduel, au-delà du niveau de protection des ouvrages, pour retenir des actions de sensibilisation, information, consultation de la population, et indique que la priorité a été donnée aux mesures non structurelles ainsi qu'aux mesures de laminageécrêtement des inondations.

$\mathrm{Au}$ Danemark, Sorensen et al. (2017) précisent que les cartes d'aléa, de vulnérabilité et de risque ont été élaborées par le gouvernement national, ainsi que des recommandations générales pour la mise en œuvre de la DI. Vingt plans de gestion ont ensuite été mis au point par les 22 municipalités concernées. Un premier bilan de l'application de la directive met en évidence la nécessité d'introduire de meilleurs systèmes d'aide à la décision, le besoin de définir des risques acceptables et l'importance d'améliorer la coordination entre les acteurs municipaux et les autres acteurs concernés, dont la société civile.

Radulescu et al. (2017) indiquent qu'en Roumanie le choix a été fait de produire un catalogue national de mesures structurelles et non structurelles pour la réduction des effets des inondations. Celui-ci a ensuite été décliné - adapté au niveau des autorités de gestion des bassins versants pour proposer des mesures spécifiques au contexte local. Ramsbottom et al. (2017) témoignent d'un appui technique réalisé en Moldavie, avec un financement de la Banque Européenne d'Investissement, pour élaborer un programme de gestion du risque d'inondation à court - moyen et long terme (respectivement échéances 1-7 ; 8-12;13-20 ans).

Neuhold (2017b) présente les principales leçons tirées d'un atelier spécifique organisé à Vienne en avril 2016 sur l'application de la DI, basé sur l'exploitation d'un questionnaire rempli 
par 28 états membres. Il en ressort qu'un progrès significatif a été accompli en termes de recensement des inondations passées et des dommages associés. La question de la gestion des incertitudes sur la cartographie des risques reste encore délicate et nécessitera des échanges - retours d'expérience entre pays. Il est encore trop tôt pour juger de l'efficacité et de l'impact des plans de gestion du risque inondation produits au cours du premier cycle d'application de la DI. On peut tout de même indiquer que la Directive a permis d'orienter les politiques de prévention vers une gamme plus large d'actions qu'auparavant (au-delà des seules mesures de protection) et que les échanges et discussions entre les différents acteurs concernés par la gestion du risque d'inondation ont progressé.

\section{RÉFÉRENCES}

Dupray S., Lavallart C. Salles E. Vignasse I. (2017) - Lessons learned from the preparation of the flood risk management plans on the Seine basin and perspectives. La Houille Blanche, 4, 13-19
Morel M., Basin B. Vullierme E. (2017) - French national policy for flood risk management. La Houille Blanche, 4, 9-12

Neunold E. (2017a) - EU Floods Directive implementation in Austria. La Houille Blanche, 4, 26-30

NeuHoLd. (2017b) - Flood Risk Management in the European Union. La Houille Blanche, 4, 53-55

Radulescu D., Ion M.B., Dumitrache R., Barbu C.E. (2017) Flood risk management objectives and Romanian catalogue of potential measures for flood prevention, protection and mitigation. La Houille Blanche, 4, 40-45

Ramsbottom D., Frank E., Weisberger A., Beros M. (2017) Developing a national programme of flood risk management measures: Moldavia. La Houille Blanche, 4, 46-52

Sorensen C., Jebens M., Piontkowitz T (2017) - Danish risk management plans of the EU Floods Directive. La Houille Blanche, 4, 31-39

Verbeke S., Devroede N. (2017) - Flood risk management in Flanders: from flood risk objectives to appropriate measures through state assessment. La Houille Blanche, 4, 20-25 